\section{NEW ATTITUDE PENALTY FUNCTIONS FOR SPACECRAFT OPTIMAL CONTROL PROBLEMS}

\begin{abstract}
Hanspeter Schaub ${ }^{*}$, Rush D. Robinett ${ }^{\dagger}$ and John L. Junkins ${ }^{\ddagger}$
To avoid the dependency of spacecraft optimal control problem solutions on the choice of attitude coordinates, a universal attitude penalty function $g()$ is presented. This function will return the same penalty for a given attitude regardless of the choice of attitude coordinates used to describe this attitude. The only singularities the $g($ ) function might encounter are solely due to the choice of attitude coordinates.

A second attitude penalty function is presented which depends on the modified Rodriguez parameters. This penalty function is simpler than the $g$ () function, but is also globally non-singular and is simpler to implement in most applications.
\end{abstract}

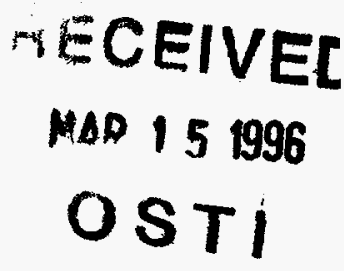

TECEIVEL OSTI

\title{
INTRODUCTION
}

A solution of a spacecraft optimal control problem, whose cost function relies on an attitude description, usually depends on the choice of attitude coordinates used. A problem could be solved using 3-2-1 Euler angles or using classical Rodriguez parameters and yield two different "optimal" solutions, unless the performance index in invariant with respect to the attitude coordinate choice. Another problem arising with many attitude coordinates is that they have no sense of when a body has tumbled beyond $180^{\circ}$ from the reference attitude. In many such cases it would be easier (i.e. cost less) to let the body complete the revolution than to force it to reverse the rotation and return to the desired attitude.

This paper develops a universal attitude penalty function $g()$ whose value is independent of the attitude coordinates chosen to represent it. Furthermore, this function will achieve its maximum value only when a principal rotation of $\pm 180^{\circ}$ from the target state is performed. This will implicitly permit the $g()$ function to sense the shortest rotational distance back to the reference state.

An attitude penalty function which depends on the Modified Rodriguez Parameters (MRP) will also be presented. These recently discovered MRPs ${ }^{1-6}$ are a non-singular three-parameter set which can describe any three-dimensional attitude. This MRP penalty function is simpler than the attitude coordinate independent $g$ () function, but retains the useful property of avoiding lengthy principal rotations of more than $\pm 180^{\circ}$.

\footnotetext{
- Graduate Research Assistant, Aerospace Engineering Department, Texas A\&M University, College Station TX 77843.

† Research Engineer, Sandia National Laboratories, Albuquerque, NM 87185.

₹ George Eppright Chair Professor of Aerospace Engineering, Aerospace Engineering Department, Texas A\&M University, College Station TX 77843, Fellow AAS.
}

This work was supported by the

United States Department of Energy under Confract DE-AC04-94AL85000. 


\section{DESCLAMITR}

Portions of this document may be illegible in electronic image products. Images are produced from the best available original document. 


\section{PROBLEM STATEMENT}

\section{Optimal Control Problem}

Most spacecraft optimal attitude control problems have a cost function $J$ which depends on the control effort, the body angular velocity and the attitude. Let $\vec{u}$ be the control torque vector, $\bar{\omega}$ be the body angular velocity vector and $\bar{\eta}$ be a generic attitude coordinate vector in the following general cost function.

$$
J=\underbrace{\frac{1}{2} K_{1} g\left(\vec{\eta}_{t_{f}}\right)+\frac{1}{2} \vec{\omega}_{t_{f}}^{T} K_{2} \vec{\omega}_{t_{f}}}_{h\left(t_{f}\right)}+\frac{1}{2} \int_{0}^{t_{f}}\left(K_{3} g(\vec{\eta})+\vec{\omega}^{T} K_{4} \vec{\omega}+\vec{u}^{T} R \vec{u}\right) d t
$$

The weights $K_{1}$ and $K_{3}$ are scalars, the weights $K_{2}, K_{4}$ and $R$ are $3 \times 3$ matrices. The spacecraft equations of motion are given in Eq. $(2,3)$ below, where the function $f(\vec{\eta})$, obtained from kinematic analysis, returns a matrix depended on the choice of attitude coordinates. The equations of motion are

$$
\begin{gathered}
\dot{\bar{\eta}}=f(\vec{\eta}) \vec{\omega} \\
\Im \dot{\bar{\omega}}=-[\tilde{\omega}] \Im \vec{\omega}+\vec{u}
\end{gathered}
$$

The matrix $\mathfrak{I}$ is the spacecraft inertia matrix. The tilde matrix is the cross-product operator

$$
[\tilde{\omega}]=\left[\begin{array}{ccc}
0 & -\omega_{3} & \omega_{2} \\
\omega_{3} & 0 & -\omega_{1} \\
-\omega_{2} & \omega_{1} & 0
\end{array}\right]
$$

The Hamiltonian $H$ for this system is

$$
H=\frac{1}{2} K_{3} g(\vec{\eta})+\frac{1}{2} \vec{\omega}^{T} K_{4} \vec{\omega}+\frac{1}{2} \vec{u}^{T} R \vec{u}+\vec{\Lambda}_{1}^{T} f(\vec{\eta}) \vec{\omega}+\vec{\Lambda}_{2}^{T} \mathfrak{I}^{-1}(-[\tilde{\omega}] \Im \vec{\omega}+\vec{u})
$$

The costate equations are given by

$$
\begin{gathered}
\dot{\Lambda}_{1}=-\frac{\partial H}{\partial \vec{\eta}}=-\frac{1}{2} K_{3} \frac{\partial g}{\partial \bar{\eta}}-\frac{\partial}{\partial \vec{\eta}}(f(\vec{\eta}) \vec{\omega}) \vec{\Lambda}_{1} \\
\dot{\Lambda}_{2}=-\frac{\partial H}{\partial \vec{\omega}}=-K_{4} \bar{\omega}-f(\vec{\eta}) \vec{\Lambda}_{1}-(\mathfrak{I}[\tilde{\omega}]-[\tilde{\mathfrak{S}}]) \mathfrak{S}^{-1} \vec{\Lambda}_{2}
\end{gathered}
$$

For unbounded control torque $\vec{u}$, the optimality condition $\partial H / \partial \vec{u}=0$ leads to the following optimal control torque

$$
\vec{u}=-R^{-1} \mathfrak{I}^{-1} \vec{\Lambda}_{2}
$$

The transversality conditions for a free final state are

$$
\begin{gathered}
\vec{\Lambda}_{1}\left(t_{f}\right)=\frac{\partial h}{\partial \bar{\eta}}\left(t_{f}\right)=\frac{1}{2} K_{1} \frac{\partial g}{\partial \vec{\eta}}\left(t_{f}\right) \\
\vec{\Lambda}_{2}\left(t_{f}\right)=\frac{\partial h}{\partial \bar{\omega}}\left(t_{f}\right)=K_{2} \vec{\omega}\left(t_{f}\right)
\end{gathered}
$$


Given good estimates of initial conditions, this nonlinear optimal control problem can be solved using various standard techniques.

\section{Attitude Coordinates}

Attitude coordinates define the rotational orientation of a body relative to some reference frame. Just as there are a number of different coordinates to describe translation (cartesian, cylindrical, spherical), there are an infinite number of different ways to described an attitude. Common examples are the Euler angles, the classical Rodriguez parameters and the Euler parameters (quaternions). Describing a rotation differs though from describing a translation. The largest difference between two rotations corresponds to a principal rotation of $\pm 180^{\circ}$, a finite value. Whereas the difference in two positions can can grow to infinity.

All minimal three-coordinate attitude representations contain a singularity. This is a specific attitude at which the coordinates are not defined. This singularity can be avoided by using the Euler parameters at the cost of adding another coordinate. This redundant set has an equality constraint which states that the attitude vector must always have unit magnitude. Therefore, if Euler parameters are used in a simple optimal control problem without any constraints, an equality constraint is automatically added.

In particular this paper will use the very elegant set of recently developed Modified Rodriguez Parameters (MRP) with their "shadow" counterpart 1,3,4. They are a non-singular, minimal coordinate representation of rigid body attitudes with several useful attributes. They can be defined as a transformation from the Euler parameters as

$$
\sigma_{i}=\frac{\beta_{i}}{1+\beta_{0}} \quad i=1,2,3
$$

or in terms of the principal rotating axis $\hat{e}$ and the principal rotating angle $\phi$ as

$$
\ddot{\sigma}=\hat{e} \cdot \tan \phi / 4
$$

Like the Euler parameters, the modified Rodrigues parameters are not unique. A second set of modified Rodrigues parameters, called the "shadow" set, can be used to avoid the singularity at the cost of a discontinuity at a switching point. The transformation between the "original" and "shadow" sets of MRPs is $1,3,4$

$$
\sigma_{i}^{S}=-\sigma_{i} / \bar{\sigma}^{T} \bar{\sigma} \quad i=1,2,3
$$

Keep in mind that the choice in distinguishing "original" and "shadow" set is purely arbitrary. both sets describe the same physical orientation. In this study the switching condition was chosen to be $\bar{\sigma}^{T} \vec{\sigma}=1$. This causes the magnitude of the orientation vector to be bounded between $0 \leq \vec{\sigma} \leq 1$ and the principal rotation angle to be restricted between $-180^{\circ} \leq \phi \leq+180^{\circ}$. Note that this combined set of "original" and "shadow" parameters implicitly "knows" the shortest way back to the origin ${ }^{1}$. Principal rotations of more than $180^{\circ}$ are typically avoided.

The differential kinematic equations of motion in terms of the modified Rodrigues parameters are given below ${ }^{1,2,6}$. The equation only contains second order polynomial nonlinearities in $\vec{\sigma}$. 


$$
\frac{d \vec{\sigma}}{d t}=\frac{1}{2}\left[I\left(\frac{1-\bar{\sigma}^{T} \bar{\sigma}}{2}\right)+[\tilde{\sigma}]+\bar{\sigma} \bar{\sigma}^{T}\right] \bar{\omega}=f(\bar{\sigma}) \vec{\omega}
$$

Eq. (14) holds for both the "original" and the "shadow" set. This means that the derivative is well defined even at the switching point. The direction cosine matrix in terms of the modified Rodrigues parameters is $1,2,6$

$$
\left.C(\bar{\sigma})=\frac{1}{\left(1+\vec{\sigma}^{T} \vec{\sigma}\right)^{2}}\left[\begin{array}{ccc}
4\left(\sigma_{1}^{2}-\sigma_{2}^{2}-\sigma_{3}^{2}\right)+\Sigma^{2} & 8 \sigma_{1} \sigma_{2}+4 \sigma_{3} \Sigma & 8 \sigma_{1} \sigma_{3}-4 \sigma_{2} \Sigma \\
8 \sigma_{1} \sigma_{2}-4 \sigma_{3} \Sigma & 4\left(-\sigma_{1}^{2}+\sigma_{2}^{2}-\sigma_{3}^{2}\right)+\Sigma^{2} & 8 \sigma_{2} \sigma_{3}+4 \sigma_{1} \Sigma \\
8 \sigma_{1} \sigma_{3}+4 \sigma_{2} \Sigma & 8 \sigma_{2} \sigma_{3}+4 \sigma_{1} \Sigma & 4\left(-\sigma_{1}^{2}-\sigma_{2}^{2}+\sigma_{3}^{2}\right)+\Sigma^{2} \\
\Sigma=1-\vec{\sigma}^{T} \vec{\sigma} & &
\end{array}\right] 15\right)
$$

In Ref. 1 it was shown that the MRP are a particular subset of a larger class of minimal attitude parameters called the Stereographic Parameters (SP). All these SP have a corresponding "shadow set" which can usually be used to avoid a singularity. However, these general SP allow the singularity to be chosen to occur at any particular principal rotation angle or about any particular principal rotation axis and angle.

\section{UNIVERSAL ATTITUDE PENALTY FUNCTION}

A scalar attitude penalty function is sought which is independent of the choice of attitude coordinates. This allows for a universal solution to many spacecraft optimal control problems. We introduce the following positive measure of attitude displacement from a reference orientation.

$$
g([C])=\frac{1}{4}(3-\operatorname{trace}([C])) \quad \in \mathfrak{R}^{+}
$$

Such a penalty function is given in Eq. (16) in terms of a proper orthogonal rotation matrix [C]. This rotation matrix is the most fundamental way to describe a rotation; unfortunately, also the most redundant. If there is no rotational displacement, the $[C]$ matrix is the identity matrix and $g([C])=0$. The largest difference between two attitudes is a principal rotation of $\pm 180^{\circ}$. In this case the $[C]$ matrix is a diagonal matrix with two entries being -1 and one being +1 . In this case $g([C])=1$. Therefore the $g()$ function is bounded for all possible motion between

$$
0 \leq g() \leq 1
$$

This penalty function can be written explicitly in terms of the principal rotation angle $\phi$ as

$$
g(\phi)=\sin ^{2}\left(\frac{\phi}{2}\right)
$$

This description makes the bound in Eq. (17) very easy to understand. The attitude cost is the highest only if the body is turned $\pm 180^{\circ}$ from the reference state.

The $g$ () function is plotted relative to the principal rotation angle $\phi$ in Figure 1. Using this type of attitude penalty function will typically avoid lengthy rotations. It intrinsically lowers the cost once the attitude has moved beyond $\pm 180^{\circ}$. 

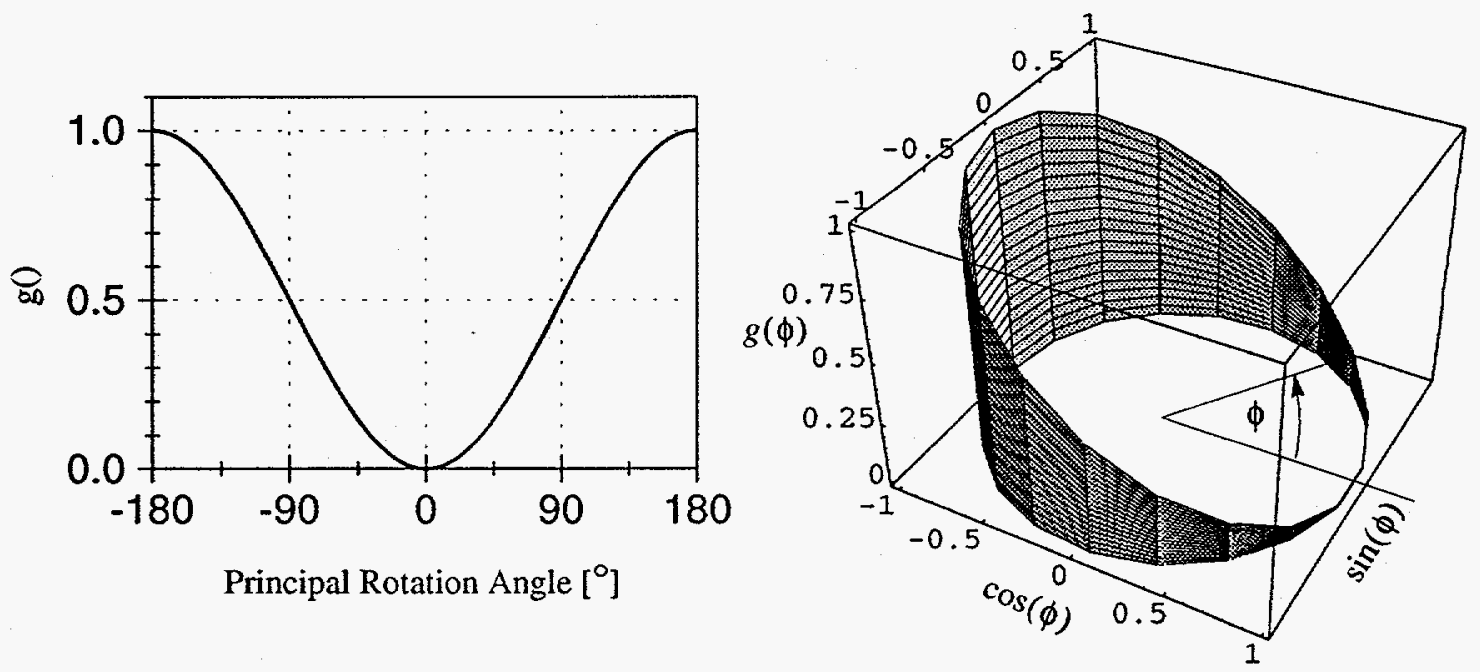

Figure 1: Illustration of the Universal Attitude Penalty Function $g()$

The advantage of defining the $g($ ) function initially in terms of the $[C]$ matrix is that this rotation matrix can be parameterized by any attitude coordinates and thus making it universally valid for any choice of attitude coordinates. The following are some sample parameterizations. Let $\bar{\beta}$ be an Euler parameter vector, then

$$
g(\vec{\beta})=\frac{1}{4}\left(3+\beta_{1}^{2}+\beta_{2}^{2}+\beta_{3}^{2}\right)
$$

and

$$
\frac{\partial g}{\partial \bar{\beta}}=\frac{1}{2}\left[\begin{array}{llll}
0 & \beta_{1} & \beta_{2} & \beta_{3}
\end{array}\right]^{T}
$$

The Euler parameters are a non-singular once-redundant set of attitude coordinates. Their drawback for optimization problems is that the redundancy introduces an additional equality constraint on the attitude vector. This redundancy also requires care to avoid other numerical problems when inverting some sets of equations.

Another popular attitude coordinate set is the classical Rodriguez parameter vector $\bar{q}$. It parameterizes the $g()$ function as

$$
g(\vec{q})=\frac{1}{4} \frac{\vec{q}^{T} \vec{q}}{1+\vec{q}^{T} \vec{q}}
$$

and

$$
\frac{\partial g}{\partial \vec{q}}=\frac{\vec{q}}{2} \frac{1}{\left(1+\vec{q}^{T} \vec{q}\right)^{2}}
$$

These coordinates are a minimal three coordinate set and don't have any problems with redundancies. However, like most three-parameters sets, they contain a singular orientation. The classical Rodriguez parameters go singular for any principal rotation of $\pm 180^{\circ}$. If it is apriori known 
that no such rotations will be encountered, this choice for attitude coordinates does allow for a large range of possible rotations.

Probably the most popular choice of attitude coordinates are any one of the 12 sets of Euler angles. Let the $\left(\theta_{1}, \theta_{2}, \theta_{3}\right)$ vector be the set of 3-1-3 Euler angles. They would parameterize the $g$ () function as

$$
g(\vec{\theta})=\frac{1}{4}\left(3-\left(1+\cos \theta_{2}\right) \cos \left(\theta_{1}+\theta_{3}\right)-\cos \theta_{2}\right)
$$

and

$$
\frac{\partial g}{\partial \bar{\theta}}=\frac{1}{4}\left[\begin{array}{l}
\left(1+\cos \theta_{2}\right) \sin \left(\theta_{1}+\theta_{3}\right) \\
\sin \theta_{2} \cos \left(\theta_{1}+\theta_{3}\right)+\sin \theta_{2} \\
\left(1+\cos \theta_{2}\right) \sin \left(\theta_{1}+\theta_{3}\right)
\end{array}\right]
$$

The advantage of the Euler angles is that they are easy to visualize, especially for small angles. However, any attitude description with Euler angles is never more than $90^{\circ}$ away from a singularity. This makes these coordinates difficult to use for large arbitrary rotations. Further, the kinematic equations for the Euler angles are in terms of trigonometric functions, making them more computationally intensive than having only polynomial equations.

A very attractive attitude description are the modified Rodrigues parameters. They parameterize the $g()$ function as

$$
g(\vec{\sigma})=4 \frac{\vec{\sigma}^{T} \vec{\sigma}}{\left(1+\vec{\sigma}^{T} \vec{\sigma}\right)^{2}}
$$

and

$$
\frac{\partial g}{\partial \vec{\sigma}}=8 \vec{\sigma}\left(\frac{1-\vec{\sigma}^{T} \bar{\sigma}}{\left(1+\bar{\sigma}^{T} \bar{\sigma}\right)^{3}}\right)
$$

The MRPs are a minimal attitude description which is also non-singular when combined with their "shadow set." They are well suited to describe any large and/or arbitrary rotation while their equations retain a simple polynomial form.

The $g()$ attitude penalty function could be parameterized by any other attitude coordinate description. Eqs. $(19,21,23,25)$ all return the same penalty for a given attitude error. This effectively removes the dependency of the optimal control solution on the choice of attitude coordinates. However, the optimal costate vector $\vec{\Lambda}_{1}$ will depend on the attitude coordinates used. Eq. (6) and (9) both depend on the partial derivative of $g()$ with respect to the particular attitude coordinates.

\section{ATTITUDE PENALTY FUNCTION USING MRP}

While the universal attitude penalty function $g()$ has some very appealing properties, it is usually more complicated than just using the standard sum squared of the attitude coordinates typically seen as an optimal control performance measure. Using the simpler attitude penalty function

$$
\stackrel{\circ}{g}(\vec{\sigma})=\vec{\sigma}^{T} \vec{\sigma}
$$


where $\vec{\sigma}$ is a MRP vector, retains all properties of the $g()$ defined in Eq. (15), except being universal with respect to attitude coordinate choice. By switching between "original" and "shadow" MRP on the $\vec{\sigma}^{T} \vec{\sigma}=1$ surface, the attitude penalties in Eq. (27) also bounded within [0,1]. Using Eq. (12) the penalty function can be written in terms of the principal rotation angle $\phi$ as

$$
\stackrel{g}{g}(\phi)=\tan ^{2}\left(\frac{\phi}{4}\right)
$$

The $\stackrel{g}{g}()$ function is plotted relative to the principal rotation angle $\phi$ in Figure 2 below. Note that like the universal attitude penalty function $g()$, the maximum attitude penalty is also attained at a principal rotation of $\pm 180^{\circ}$.
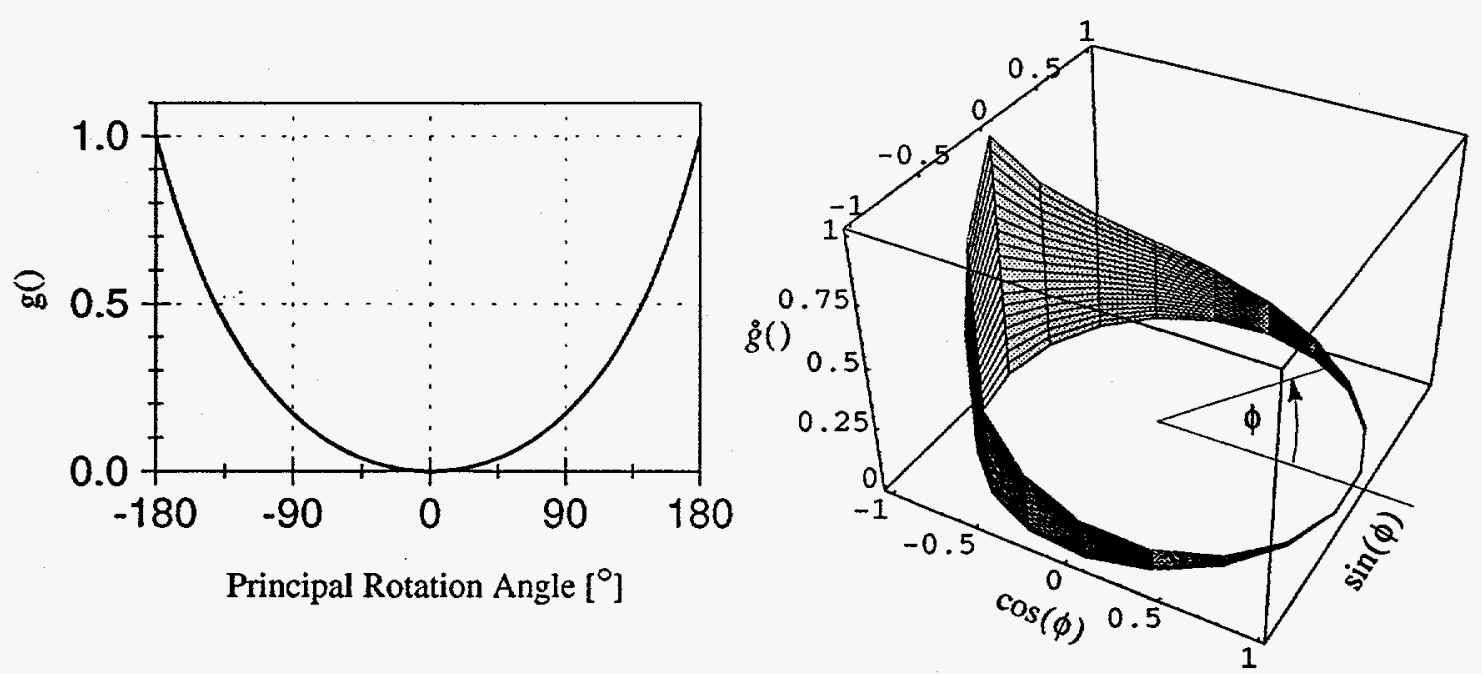

Figure 2: Illustration of the attitude penalty function $g($ )

By using the MRPs, this penalty function is globally non-singular using a minimal attitude coordinate description. The non-singularity comes at the price of having the switching condition defined in Eq. (13). However, both $\bar{\sigma}$ and $\dot{\sigma}$ are well defined for both the original and the "shadow" parameters. By choosing the switching surface $\vec{\sigma}^{T} \vec{\sigma}=1$ Eq. (13) gets simplified to

$$
\vec{\sigma}^{s}=-\vec{\sigma}
$$

and the derivative of the "shadow" trajectory at the switching point is

$$
\dot{\bar{\sigma}}^{S}=\dot{\bar{\sigma}}+2[\bar{\omega}] \bar{\sigma}
$$

Observe from Eq. (30) that for pure single axis rotations $\dot{\bar{\sigma}}$ simply equals $\dot{\bar{\sigma}}^{S}$ on the switching surface. Since the derivatives of the costates depend on the attitude coordinates, they will also have a discontinuity as the attitude vector is switched. Using Eqs. (14) and (18), the costate differential equations can be written in terms of the MRP as

$$
\begin{gathered}
\dot{\bar{\Lambda}}_{1}=-K_{1} \vec{\sigma}+\frac{1}{2}\left(\bar{\sigma} \vec{\omega}-\vec{\omega}-\vec{\omega} \vec{\sigma}^{T}-I \cdot \vec{\sigma} \vec{\omega}\right) \vec{\Lambda}_{1} \\
\dot{\bar{\Lambda}}_{2}=-K_{2} \vec{\omega}-\frac{1}{2}\left(\frac{1-\bar{\sigma}^{T} \vec{\sigma}}{2} I-[\tilde{\sigma}]+\vec{\sigma} \vec{\sigma}^{T}\right) \vec{\Lambda}_{1}-(\mathfrak{I}[\tilde{\omega}]-[\tilde{I} \omega]) \Im^{-1} \vec{\Lambda}_{2}
\end{gathered}
$$




\section{RESULTS}

\section{Universal Attitude Penalty Function}

To illustrate the use of the universal attitude penalty function, a simple one-dimensional optimal control problem is considered. The spacecraft initial conditions are $\phi(0)=165^{\circ}$ and $\omega(0)=$ $80 \%$. Since there is a large positive initial angular velocity, it would make intuitive sense to let body complete the revolution and then bring it to rest. If only the square of the angle $\phi$ were used as the attitude penalty function, such a maneuver would not be possible. Rotating past $180^{\circ}$ would yield an ever increasing attitude penalty. Thanks to the properties of the $g$ () function however, the attitude penalty will get reduced automatically after rotating through $180^{\circ}$.

The cost weights used were $K_{1}=3, K_{2}=5, K_{3}=6, K_{4}=10$ and $R=20$. This weights will force the optimization to not only minimize the end states, but also penalize the torque and state variations to get there. The inertia was set to $1 \mathrm{kgm}^{2}$. The optimal control problem was solved numerically using a gradient algorithm with 1500 integration steps. The MRP were used as attitude coordinates. However, the attitude output in Figure 1 was transformed back into the principal rotation angle for easier visualization.

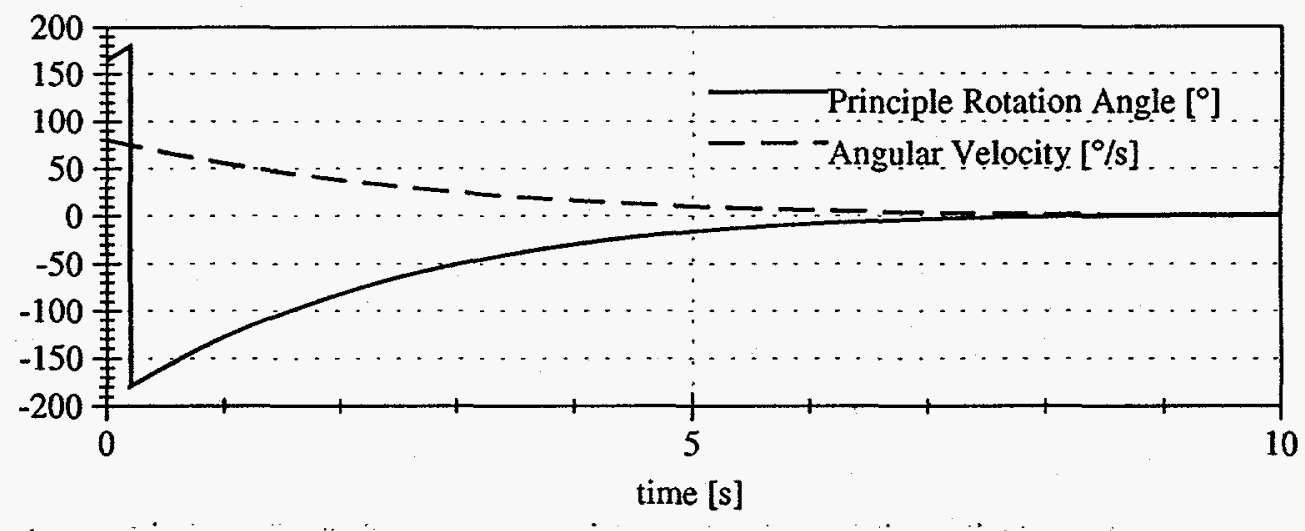

Figure 1: Optimal states using the $g()$ function

The optimal solution found did indeed let the spacecraft rotate on past $180^{\circ}$ and slowed it down when it completed the revolution. The angular velocity also gets smoothly reduced as the body slowly comes to a halt at $\phi=0^{\circ}$.

The costate equations are shown in Figure 2. Since the MRP with their "shadow counterparts" were used as attitude parameters, the attitude has a discontinuity as the spacecraft rotates through the $180^{\circ}$ heading. Since $g()$ is universal, there is no discontinuity at the switching points where $\bar{\sigma}^{T} \vec{\sigma}=1$. Since the derivative of the costate $\vec{\Lambda}_{1}$ depends of the partial derivative of $g()$ with respect to the attitude parameters, it too will have a discontinuity when the MRP are switched. The resulting corner in $\vec{\Lambda}_{1}$ is clearly visible in Figure 2 . Since the costate $\vec{\Lambda}_{2}$ is independent of the choice of attitude coordinates, it does not display any corner during the MRP switching. 


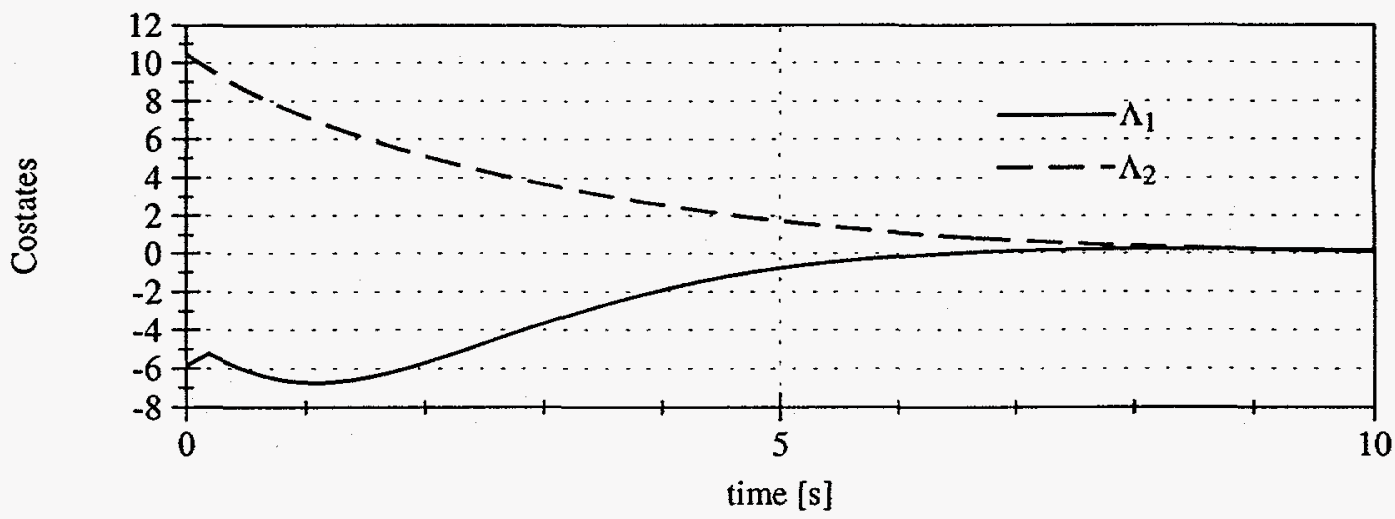

Figure 2: Optimal Costates using the $g($ ) function

The optimal control torque is shown in Figure 3. Note that the torque is always negative in this case. This means the control is only "braking" the system and never speeding it up in another direction, making the control effort more efficient.
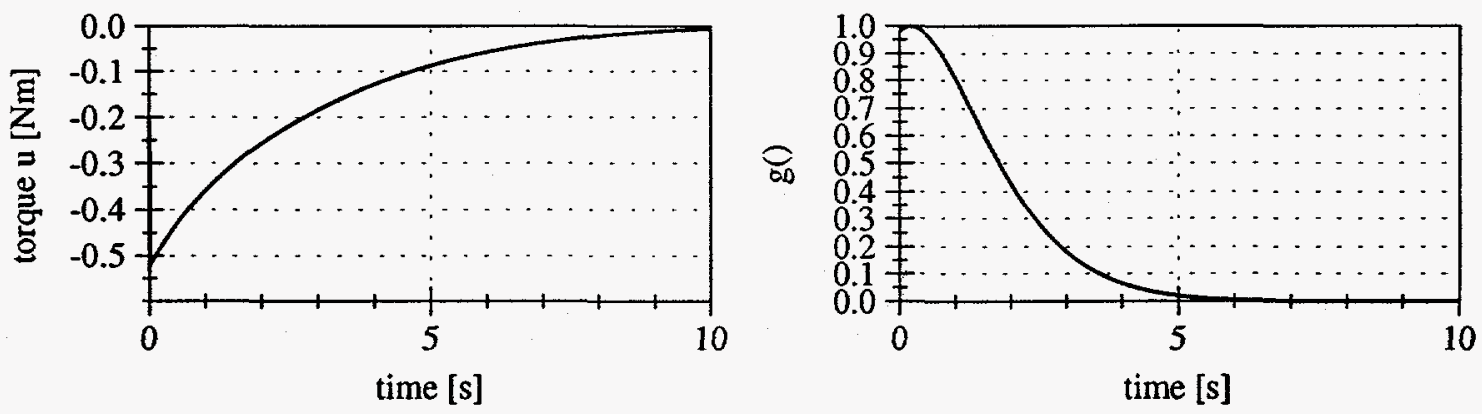

Figure 3: Optimal Control Torque and Attitude Penalty Function

The time history of the universal attitude penalty function $g()$ is also shown in Figure 4 . Since the initial angle is very close to $180^{\circ}$, the initial attitude penalty is almost 1. As the maneuver is completed the attitude penalty quickly drops to around zero. Recall that $g()$ is only a portion of the performance index of Eq. (1).

\section{MRP Attitude Penalty Function}

This optimization problem used the same spacecraft with identical initial states as the previous simulation. This time the attitude penalty function defined in Eq. (26) was used. Only the weights $K_{1}$ and $K_{3}$ were changed to 2 and 1 respectively (since the attitude penalty function has changed), to make the maneuvers qualitatively similar.

The optimal states obtained using the MRP attitude penalty function $\stackrel{g}{(0)}$ are shown in Figure 4. This cost function also allowed the spacecraft to complete the revolution rather than making it reverse its angular velocity. The optimal states for this simulation are only qualitatively comparable to the ones from the previous simulation since a different attitude penalty function and weights were used. 


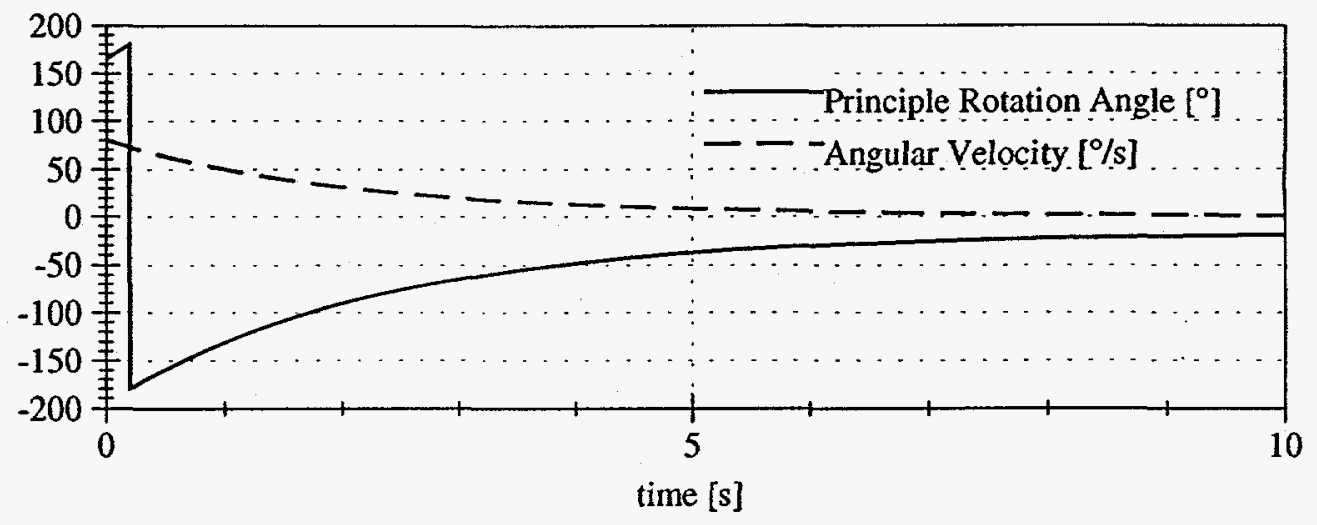

Figure 4: Optimal States using the $\stackrel{g}{g}()$ function

The optimal costates are shown in Figure5. Note that the time history of $\Lambda_{\mathbf{1}}$ differs considerably from the previous simulation.

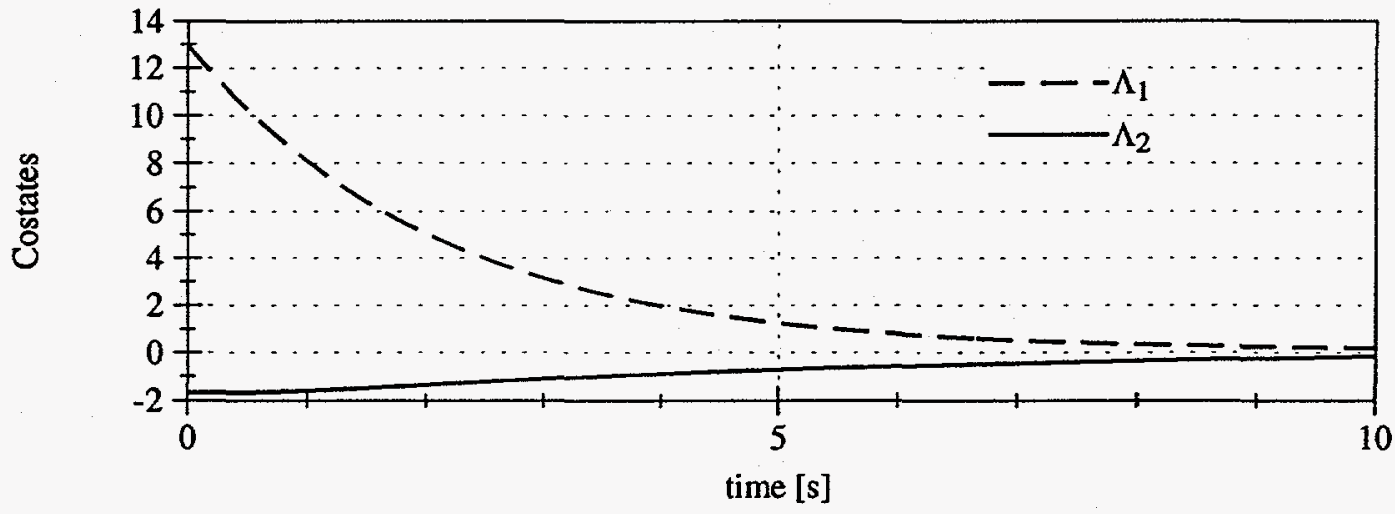

Figure 5: Optimal Costates using the $\stackrel{g}{g}()$ function

- The optimal control and the optimal attitude penalty function $g()$ are shown in Figure 6 . The control torque is also only used to brake the system, never to speed it up.
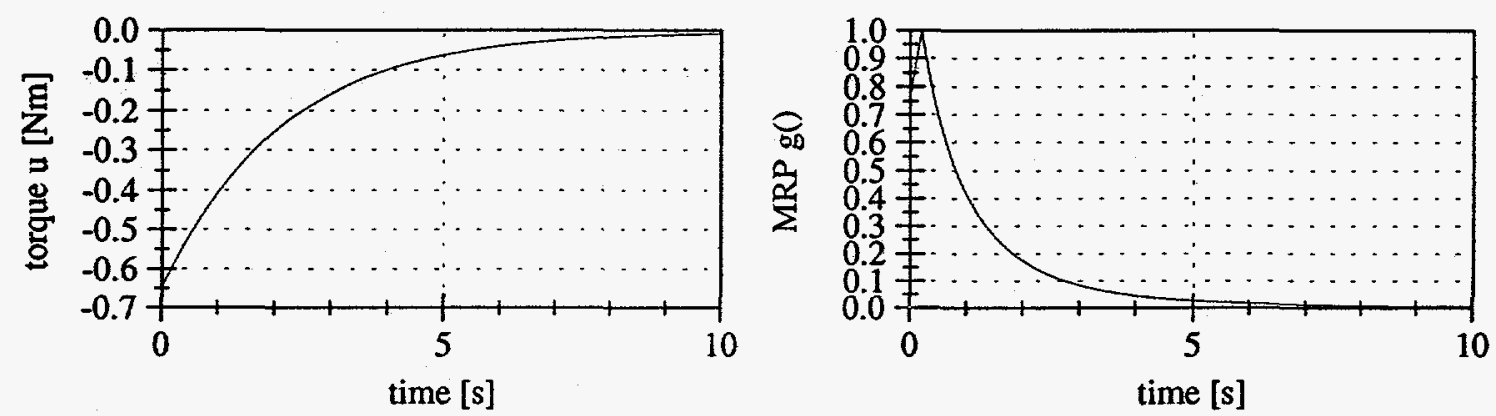

Figure 6: Optimal Control Torque and Attitude Penalty Function

Note the difference in the attitude penalty function around the $180^{\circ}$ heading. This penalty function reaches its maximum much more sharply than did the $g($ ) function. 


\section{CONCLUSION}

A universal attitude penalty function $g($ ) was presented which allows for optimal spacecraft control solutions which do not depend on the choice of attitude parameters. This penalty function is non-singular. Nevertheless, depending on what attitude coordinates are used to describe $g()$, the parameterization of $g()$ could contain some singular attitudes. However, either the Euler parameters or the MRP could be used to provide a non-singular description. The $g($ ) function also has another useful property in that it doesn't penalize rotation past $180^{\circ}$.

A second attitude penalty function was presented that depended on the use of the MRP. This non-universal function is of simpler form that the $g($ ) function, but retains the most useful attributes. It is also globally non-singular and doesn't penalize rotations through $180^{\circ}$.

\section{REFERENCES}

[1] Schaub, H., and Junkins, J. L., "Stereographic Orientation Parameters for Attitude Dynamics: A Generalization of the Rodrigues Parameters," AAS/AIAA Spaceflight Mechanics Meeting, Albuquerque, New Mexico, Feb. 13-16, 1995, paper AAS 95-137.

[2] Tsiotras, P. "New Control Laws for the Attitude Stabilization of Rigid Bodies," Proceedings, IFAC Symposium on Automatic Control in Aerospace, Palo Alto, CA, Sept. 12-16, 1994, pp. 316-321.

[3] Schaub, H., Tsiotras, P., and Junkins, J. L., "Principal Rotation Representations of Proper NxN Orthogonal Matrices," to appear in International Journal of Engineering Science, 1994.

[4] Marandi, S. R., and Modi, V. J., "A Preferred Coordinate System and the Associated Orientation Representation in Attitude Dynamics," Acta Astronautica, Vol. 15, 1987, pp. 833-843.

[5] Wiener, T. F., "Theoretical Analysis of Gimballess Inertial Reference Equipment Using Delta-Modulated Instruments," Diss. Massachusetts Institute of Technology, March 1962.

[6] Shuster, M. D., "A Survey of Attitude Representations," Journal of the Astronautical Sciences, Vol. 41, No. 4, 1993, pp. 439-517.

\section{DISCLAIMER}

This report was prepared as an account of work sponsored by an agency of the United States Government. Neither the United States Government nor any agency thereof, nor any of their employees, makes any warranty, express or implied, or assumes any legal liability or responsibility for the accuracy, completeness, or usefulness of any information, apparatus, product, or process disclosed, or represents that its use would not infringe privately owned rights. Reference herein to any specific commercial product; process, or service by trade name, trademark, manufacturer, or otherwise does not necessarily constitute or imply its endorsement, recommendation, or favoring by the United States Government or any agency thereof. The views and opinions of authors expressed herein do not necessarily state or reflect those of the United States Government or any agency thereof. 